\title{
Low frequency of HER2 amplification and overexpression in early onset gastric cancer
}

\author{
Cathy B. Moelans • Anya N. Milne • \\ Folkert H. Morsink • G. Johan A. Offerhaus • \\ Paul J. van Diest
}

Accepted: 12 February 2011 / Published online: 11 March 2011

(C) The Author(s) 2011. This article is published with open access at Springerlink.com

\begin{abstract}
Background The recent ToGA trial results indicated that trastuzumab is a new, effective, and well-tolerated treatment for HER2-positive gastric cancer (GC). Although GC mainly affects older patients, fewer than $10 \%$ of GC patients are considered early-onset (EOGC) (presenting at the age of 45 years or younger). These EOGC show different clinicopathological and molecular profiles compared to late onset GC suggesting that they represent a separate entity within gastric carcinogenesis. In light of potential trastuzumab benefit, subpopulations of GC such as EOGC (versus late onset) should be evaluated for their frequency of amplification and overexpression using currently available techniques.

Methods Tissue microarray (TMA) blocks of 108 early onset GC and 91 late onset GC were stained by immunohistochemistry (IHC, Hercep test, DAKO) and chromogenic in situ hybridization (CISH, SPoT-Light, Invitrogen).

Results Overall, we found only 5\% HER2 high level amplification and 3\% HER2 3+ overexpression (6/199). In addition, 8 patients (4\%) showed a low level CISH amplification and 9 patients $(4.5 \%)$ showed a $2+$ IHC score. IHC and CISH showed $92 \%$ concordance and CISH showed less heterogeneity than IHC. In 2/199 cases $(1 \%)$, IHC showed clinically relevant heterogeneity between TMA cores, but all cases with focal IHC 3+ expression were uniformly CISH high level amplified. Early onset GCs showed a significantly lower frequency
\end{abstract}

C. B. Moelans $(\bowtie) \cdot$ A. N. Milne $\cdot$ F. H. Morsink

G. J. A. Offerhaus $\cdot$ P. J. van Diest

Department of Pathology, University Medical Center Utrecht,

Heidelberglaan 100, PO Box 85500, 3508GA Utrecht,

The Netherlands

e-mail: cmoelans@umcutrecht.nl of HER2 amplification (2\%) and overexpression ( $0 \%)$ than late onset GCs ( $8 \%$ and $7 \%$ respectively) ( $p=0.085$ and $p=0.008$ respectively). Proximal GC had more HER2 amplification $(9 \%$ versus $3 \%)$ and overexpression $(7 \%$ versus $2 \%$ ) than distal tumours although this difference was not significant ( $p=0.181$ and $p=0.182$ respectively). HER2 CISH showed more high level amplification in the intestinal type (7\%, 16\% if low-level included) compared to the mixed (5\%, 5\% if low-level included) and diffuse type $(3 \%, 4 \%$ if low-level included) GCs $(p=0.029)$. A similar association was seen for HER2 IHC and histologic type $(p=0.008)$. Logistic regression indicated a significant association between HER2 expression and age, which remained significant when adjusted for both location and histological type.

Conclusions Even focal HER2 overexpression in GC points to uniform HER2 amplification by CISH. We show for the first time that early onset GC has a lower frequency of HER2 amplification and overexpression than late onset $\mathrm{GC}$, and confirm that intestinal type GC shows the highest rate of HER2 amplification and overexpression.

Keywords HER2 - Gastric cancer - Immunohistochemistry · In situ hybridization

\section{Introduction}

Gastric cancer (GC) affects about one million people a year and is the second leading cause of cancer-related mortality worldwide [33]. GC exists as two main histological types, diffuse and intestinal, as described by Laurén et al. [20], and is thought to result from a combination of environmental factors and accumulation of specific genetic alterations. GC mainly affects older patients: fewer than $10 \%$ of 
gastric cancer patients are considered early-onset (EOGC) (presenting at the age of 45 years or younger) [19]. Most young patients present at advanced clinical stage similar to elderly patients, so the prognosis in both age groups is poor. It is postulated that genetic factors may play a more important role in EOGC as in older patients as they have less exposure to environmental carcinogens [8], thus molecular studies in these cancers could play a key role in the unravelling of genetic changes in gastric carcinogenesis. Only $10 \%$ of these young patients have a positive family history [19] which emphasises that $90 \%$ of the occurrence of EOGC remains largely unexplained. The clinicopathological features of GC differ between young and elderly patients. Young patients have a female preponderance, are more often multifocal [10], more frequently develop diffuse lesions and less frequently develop intestinal metaplasia $[19,21,24]$. The predominance of females is considered by some to be due to hormonal factors [22]. Furthermore, previous studies have shown a different molecular expression profile in early-onset GC (EOGC) than in late-onset GC $[7,27,29]$. The fact that these EOGC show different clinicopathological and molecular profiles compared to late onset GC suggests that they represent a separate entity within gastric carcinogenesis.

Surgery is the mainstay of treatment for resectable GC, but recurrence rates are high even after curative resection. In Western countries, most patients are diagnosed at an advanced (unresectable) stage and, despite benefits of palliative radiotherapy and chemotherapy, prognosis of advanced GC remains poor (median survival 7-10 months). Most promise to improve this poor survival is provided by biologically targeted agents. The ToGA study was the first randomized, prospective, multicenter, phase III trial to evaluate the efficacy and safety of trastuzumab (Herceptin ${ }^{\mathrm{TM}}$ ), a humanized monoclonal anti-HER2 antibody treatment, in HER2-positive GC $[14,16]$. The modest but clinically significant overall survival benefit indicated that trastuzumab is a new, effective, and well-tolerated treatment for HER2-positive GC. Since 1998, trastuzumab has been used to treat more than 740,000 patients with HER2positive breast cancer worldwide, so there is much to learn from the diagnostic methods developed to detect HER2 amplification and overexpression to select breast cancer patients for this treatment. At present, the most common methods to assess HER2 status in breast cancer are immunohistochemistry (IHC) and chromogenic and fluorescence in situ hybridization (CISH and FISH). CISH and IHC allow easier morphologic examination than FISH, thereby facilitating detection of heterogeneity. This is important in GC since higher rates of heterogeneity have been reported in GC (5\%) compared to breast cancer $(1.5 \%)[15]$.
This study is of interest for treatment of gastric cancer patients with trastuzumab. Since HER2 expression is more often detected in intestinal type gastric cancers compared to diffuse type gastric cancers $[2,39]$ and tumours of young patients are generally known to have more frequently diffuse type gastric cancers [32, 40], we would expect HER2 expression to be lower in young patients. However, some studies have suggested that HER2 positivity is associated with poor outcome in gastric cancer, and with more aggressive disease [12, 39, 43]. It has also been suggested, although not consistently, that young patients have more aggressive tumour behaviour [37, 40], in which case we would expect HER2 expression to be higher in young patients. Thus, the goal of this study was to assess HER2 amplification and overexpression frequencies in a large group $(n=199)$ of well documented GC, including 108 EOGC and 91 late onset GC, using HER2 testing technologies currently in use such as IHC and CISH.

\section{Materials and methods}

\subsection{Patient material}

This study involved 199 GC (of which 108 EOGC) collected for previous studies from 24 different institutions throughout The Netherlands, Poland, Finland and the USA. Anonymous use of redundant tissue for research purposes is part of the standard treatment agreement with patients in the collaborating hospitals and all blocks were obtained through the government approved nationwide PALGA database with an approved scientific protocol. Tissue microarrays were constructed from formalin-fixed paraffin embedded archival specimens. Three core biopsies $(0.6 \mathrm{~mm}$ in diameter) were taken from histologically representative regions (including heterogeneous areas) and arranged in a new recipient paraffin block. Control samples included normal tissue from gastric mucosa, liver and lymph node. Four-micrometer sections were made to perform IHC and CISH assays. From a previous study, histological grade and stage according to the TNM 5th edition (UICC 1997) were available [27]. Table 1 shows the clinicopathological data of the early versus late onset patients.

\subsection{Immunohistochemistry}

The HercepTest ${ }^{\mathrm{TM}}$ (DAKO, Glostrup, Denmark) was used according to the protocol of the manufacturer. Cell line test slides provided by the manufacturer were used as positive and negative controls. Immunostaining was scored by one pathologist (PvD) following a 4-step scale $(0,1+, 2+$ and $3+)$, according to the consensus panel recommendations on HER2 scoring for GC biopsies [15] 
Table 1 Clinicopathological data of early and late onset gastric cancer patients in this study

\begin{tabular}{|c|c|c|c|}
\hline & & $\begin{array}{l}\text { Early onset } \\
n=108\end{array}$ & $\begin{array}{l}\text { Late onse } \\
n=91\end{array}$ \\
\hline \multirow[t]{3}{*}{ HER2 } & $0 / 1+$ & 106 & 76 \\
\hline & $2+$ & 2 & 8 \\
\hline & $3+$ & 0 & 7 \\
\hline \multirow[t]{2}{*}{ Age } & Range & $21-45$ & $47-86$ \\
\hline & Median & 35 & 68 \\
\hline \multirow[t]{4}{*}{ T status } & pT1 & 8 & 6 \\
\hline & pT2 & 25 & 47 \\
\hline & pT3 & 29 & 35 \\
\hline & pT4 & 4 & 3 \\
\hline \multirow[t]{4}{*}{$\mathrm{N}$ status } & pN0 & 20 & 22 \\
\hline & $\mathrm{pN} 1$ & 29 & 43 \\
\hline & $\mathrm{pN} 2$ & 8 & 18 \\
\hline & $\mathrm{pN} 3$ & 3 & 6 \\
\hline \multirow[t]{2}{*}{ Location } & Proximal & 8 & 49 \\
\hline & Distal & 69 & 42 \\
\hline \multirow[t]{3}{*}{ Histology } & Mixed & 7 & 11 \\
\hline & Diffuse & 75 & 30 \\
\hline & Intestinal & 23 & 50 \\
\hline
\end{tabular}

with and without the additional Ruschoff criteria [34]. Scoring was done blinded to CISH results. Variation between the IHC scores of the three cores for each case (indicating intra-tumour heterogeneity) was noted, as well as the clinical relevance of these variations $(3+$ vs. lower scores). The highest of all three scores was used as the final score per tumour.

\subsection{Chromogenic in situ hybridization}

All CISH assays were performed using the Invitrogen SPoT-Light HER2 CISH kit according the manufacturers' instructions. A positive control was included in each CISH run and consisted of paraffin sections of a breast tumour known to be HER2 amplified by CISH. For scoring, the presence of large peroxidase-positive intra-nuclear clusters or $>10$ individual small signals in $>50 \%$ of tumour cells were considered amplified. The presence of small peroxidase-positive intra-nuclear clusters or 6-10 individual small signals were considered low-level amplified. Tumours with 5 or less individual small signals per nucleus were scored as non-amplified. Scoring was done in at least 20 tumour cells by one pathologist (PvD), blinded to IHC results. Variations between the CISH scores of the three cores for each case (indicating intratumour heterogeneity) was noted, as well as the clinical relevance of these variations (high-level amplified vs. low-level amplified/normal).

\subsection{Statistics}

Statistics were performed using SPSS statistical software. Contingency tables and chi-square analyses were used to analyze associations between IHC and CISH results and between both techniques and clinico-pathological features. $P$-values below 0.05 were considered significant and posthoc Bonferroni correction was used to address the problem of multiple comparisons when applicable. Binary logistic regression was used to investigate the relationship between variables.

\section{Results}

\subsection{Immunohistochemistry}

Overall, 6/199 (3\%) GC showed HER2 3+ overexpression by IHC, an additional 9/199 (4.5\%) were scored 2+, $23 / 199(11.5 \%) 1+$ and the remaining 161 tumours $(81 \%)$ were negative. None of the EOGC $(n=108)$ showed overexpression but $7 \%$ of the other 91 late onset GC were IHC $3+(p=0.008)$. The EOGCs showed IHC $2+, 1+$ and 0 scores in $2 \%, 6.5 \%$ and $91.5 \%$, respectively, versus $8 \%$, $18 \%$ and $68 \%$, respectively, in late onset GCs.

\subsection{Chromogenic in situ hybridization}

CISH failed for four tumours because of overdigestion or because less than 20 tumour cells were left for evaluation. Overall, 9/195 (5\%) GC showed HER2 high level amplification by CISH, with $8 \%$ amplification in late onset GC and only $2 \%$ in EOGC $(p=0.085)$. A low-level amplification was detected in an additional $4 \%$ of the analyzed GC, with $5 \%$ in the late onset GC group and 3\% in the EOGC group. In total, this study thereby detected $13 \%$ amplification (low plus high level) in the late onset GC group and $5 \%$ in the EOGC group $(p=0.069)$. Furthermore, although many tumours were classified as non-amplified by CISH, $31 \%$ of the late onset GCs and $12 \%$ of the EOGCs had more than 2 (but less than 6) HER2 copies.

\subsection{Correlation between IHC and CISH}

Table 2 and Fig. 1 show a 92\% concordance between IHC and CISH (180/195). Of the 15 discrepant tumours, 7 were scored IHC $0 / 1+$ while CISH was scored low-level amplified ( $3 / 7$ were EOGC), 5 were scored $2+$ by IHC but showed no CISH amplification and 3 were scored $2+$ by IHC but showed amplification by CISH (2/3 were EOGC).

Without applying Ruschoff criteria, IHC showed clinically relevant heterogeneity between the three TMA cores 
Table 2 Correlation between HercepTest immunohistochemistry and HER 2 chromogenic in situ hybridization (CISH) in 195 gastric carcinomas

\begin{tabular}{llllll}
\hline & & & & \\
\cline { 3 - 5 } & & & & & Total \\
\cline { 3 - 5 } & $1-5$ copies & $6-10$ copies & $>10$ copies & \\
\hline HercepTest & $0 / 1+$ & 173 & 7 & 0 & 180 \\
& $2+$ & 5 & 1 & 3 & 9 \\
Total & $3+$ & 0 & 0 & 6 & 6 \\
\hline
\end{tabular}

in 4/195 (2\%) cases $(2 \mathrm{x} 1+/ 3+/ \mathrm{CISH}$ high level amplified, and $2 \mathrm{x} 1+/ 2+/ \mathrm{CISH}$ high level amplified). In contrast, $\mathrm{CISH}$ did not show such heterogeneity and was stable across the three TMA cores for all cases. With Ruschoff criteria applied, the clinically relevant heterogeneity was reduced to $2 / 195$ cases $(2 \times 1+/ 2+/ C I S H$ high level amplified). In total, after rescoring according to the Ruschoff system for biopsies [34], we adjusted 21/199 scores $(15 \mathrm{x} 0$ to $1+$ and $6 \mathrm{x} 1+$ to $2+)$ in comparison to the Hofmann scoring system alone [15], thereby taking into account the three scores of all three cores per tumour (the highest of all three scores was used as the final score per tumour). Applying this new scoring system often resulted in a more uniform score across the three cores. Also, in several cases when $\mathrm{CISH}$ revealed more copies although no amplification, the IHC score became $1+$ (from 0 ) after rescoring. However, by applying these additional criteria, the concordance between IHC and CISH was reduced because of the increased number of $2+$ cases $(9$ instead of 3) without corresponding CISH amplification.

3.4 Correlations between HER2 status and clinicopathological features

Proximal GC had more HER2 amplification (9\% versus $3 \%$ ) and overexpression (7\% versus $2 \%$ ) than distal tumours but this association was not significant ( $p=$ 0.181 and $p=0.182$ respectively). HER2 CISH showed more high level amplification in the intestinal type $(7 \%$, $16 \%$ if low-level included) compared to the mixed $(5 \%$, $5 \%$ if low-level included) and diffuse type (3\%, $4 \%$ if low-level included) GCs $(p=0.029)$. A similar association was seen for HER2 IHC and histologic type $(p=0.008)$. There was a significant association between HER2 overexpression and age $(p=0.008)$ and a trend towards an association between HER2 (low plus high level) ampli-
Fig. 1 Two cases with HER2 overexpression by immunohistochemistry (IHC, HercepTest) and amplification by chromogenic in situ hybridization (CISH). a Case 1 with IHC $3+$ score and corresponding b CISH amplification. c Case 2 with IHC $3+$ score and corresponding d CISH amplification
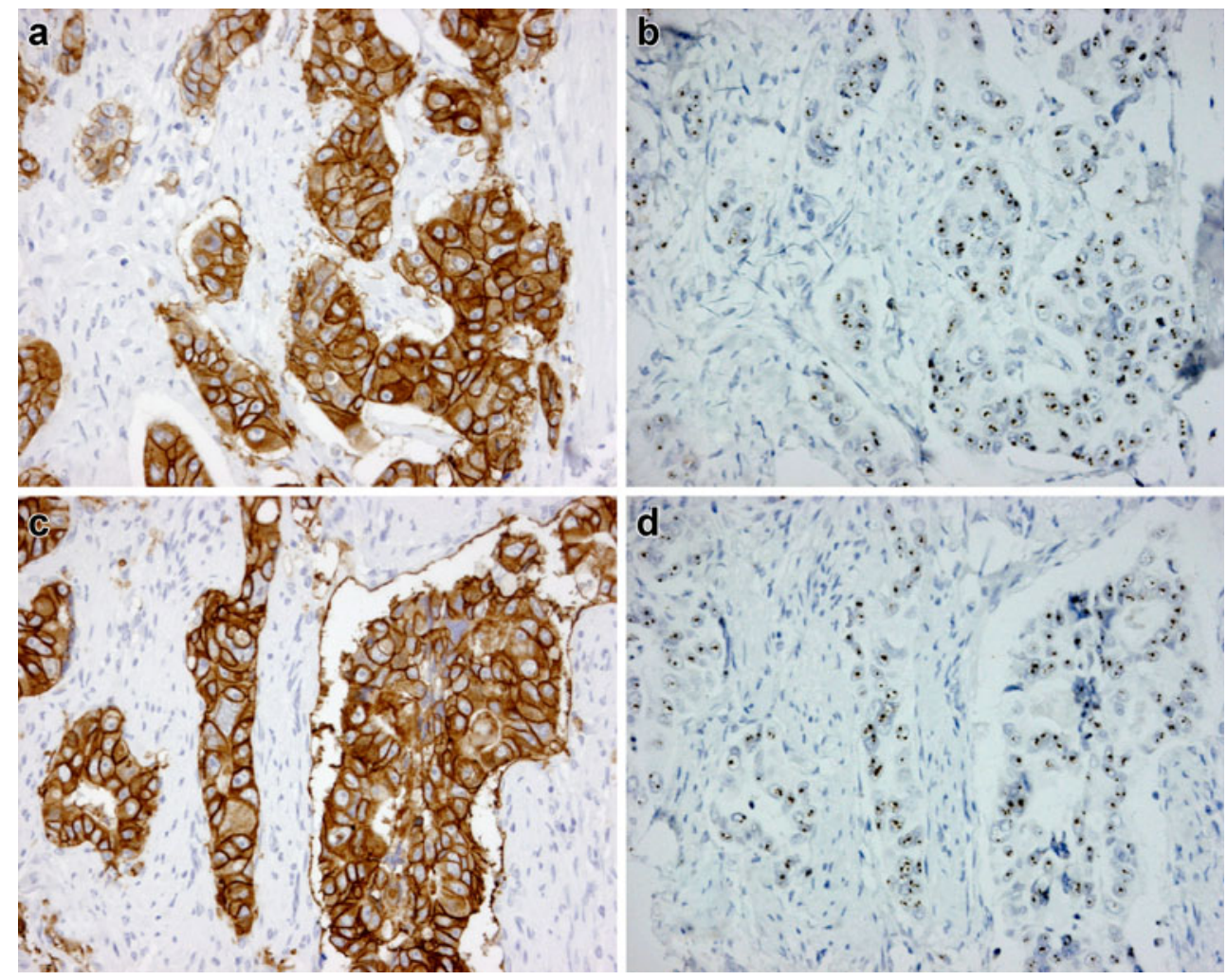
fication and age $(p=0.069)$. There was no significant correlation between HER2 amplification or overexpression levels and T- or N status.

Logistic regression indicated a significant association between HER2 expression and age, which remained significant when adjusted for both location and histological type. The significant association found between HER2 and histology type was no longer significant when age was accounted for in the regression analysis.

\section{Discussion}

This study aimed to assess HER2 amplification and overexpression frequencies in a large group $(n=199)$ of well documented GC, including 108 EOGC, using IHC and CISH. Reported rates of overexpression and amplification of HER2 in GC vary widely (2-45\%) due to small sample sizes, different study populations and methodological and scoring differences between studies $[3,11,15,23,31,39$, 44, 45]. The largest data set of $>3,800$ advanced GC samples, found HER2 positivity rates of $22.1 \%$ [2, 14, 42] and tumours classified as intestinal type were significantly more likely to be HER2-positive (32\%) than diffuse (only $6 \%$ ) or mixed (20\%) types. A recent review combining data from 24 studies $(6,542$ patients) calculated a weighted mean of $19 \%$ HER 2 positivity [16]. The present study reports frequencies in the lower range, with EOGC having an even lower percentage of amplification (2\%) and overexpression $(0 \%)$ than late onset $\mathrm{GC}$ ( $8 \%$ and $7 \%$ respectively). Other studies have shown that EOGC have a different molecular expression profile than late onset $\mathrm{GC}[26,27]$ which is consistent with the finding that these cancers show a different (lower) HER2 overexpression and amplification frequency than late onset GC. Other studies using whole slides have reported lower frequencies of overexpression as well $[3,38,41]$. A recent study used two independent data sets and performed IHC on whole slides for one of the data sets and on TMA slides for the other data set. They found $9 \%$ and $4 \%$ HER 2 positivity respectively, and suggested tumour heterogeneity and subsequent sampling error using TMA cores as the reason for this difference in expression frequency [11]. Both series also included young patients but it is unclear how many (only range and median were given, both medians were $>60$ ). Although in the present study we report low overexpression/amplification frequencies similar to Grabsch et al., we are convinced that there is a significant difference in HER2 expression between both age groups in our very large set of EOGC, underlined by the results of logistic regression analysis.

As reported in previous studies [1, 39], there was an association between HER2 amplification/overexpression, tumour location (proximal vs. distal) and tumour type (intestinal vs. diffuse vs. mixed). However, after logistic regression analysis and correction for age, these associations were no longer significant. More importantly, the present study also reports a significant association between HER 2 overexpression and older age. It has been claimed that young patients have a poorer prognosis [37, 40], but others have reported that tumour staging and prognosis for young patients is similar to older patients and depends on whether the patients undergo a curative resection $[18,19$, 24, 35]. Unfortunately this study did not include follow-up data but no relation was found between age and lymph node involvement or $\mathrm{T}$ stage (which makes prognostic correlations unlikely), nor between HER2 status and both prognostic factors.

A recent paper on breast cancer in young $(<40$ years $)$ patients could not find a significant correlation between HER 2 expression $(2+/ 3+)$ and age, and even seemed to find slightly more overexpression in younger patients $(23 \%)$ compared to a control group (18\%) [9]. However, they did find a higher frequency of triple negatives (ER, PR and HER2) in the younger group. Furthermore, in breast cancer, there does not seem to be a correlation between age of diagnosis and reponse to trastuzumab therapy. Four large trials of adjuvant trastuzumab demonstrated significant improvements in disease-free survival $(33 \%-52 \%)$ and overall survival $(34 \%-41 \%)$ independent from tumour size, nodal or hormone-receptor status, and age [5]. This indicates that gastric cancer seems to be quite different from breast cancer in terms of HER2 expression in younger patients, and further supports the increasing evidence that EOGC evolves through a different molecular genetic pathway than conventional gastric cancers $[6,7,25-29$, 36].

There are at this point some scarce reports on the association between HER2 amplification status and response to therapy in breast cancer. A very recent study, for example, showed that the level of HER2 gene amplification significantly influenced pCR but not RFS or OS in nonmetastatic breast cancer treated with trastuzumab-based neoadjuvant therapy. However, RFS in patients with highly amplified (HA) tumours tended to be shorter [13]. Results from CALGB150002 have shown a relationship between response rate to trastuzumab and HER2 copy number ratio by FISH but also suggested that trastuzumab might be effective in a subpopulation of breast cancer conventionally defined as HER2 negative (because of polysomy 17), but in fact displaying low level HER2 amplification [17]. Another study found no significant association between HER2 copy number and benefit and showed that even patients with normal HER2 gene copy numbers appeared to benefit [30]. These studies thus indicate that the level of HER2 amplification plays a role in therapy response, but also that 
patients with no or lower HER2 gene copy numbers could benefit from trastuzumab. Although detailed age information was not published, both age groups evaluated in the ToGA trial $(<60$ or $\geq 60$ years, selected based on IHC or FISH positivity) showed trastuzumab response but the overall survival of the younger category seemed to be lower (HR 0.84) than the older category (HR 0.66) in the trastuzumab plus chemotherapy arm [2]. Furthermore, this trial also showed that patients with diffuse type GC (more common in EOGC) do not benefit from the addition of trastuzumab (HR 1.07 versus HR 0.69 and HR 0.86 for intestinal and mixed histological type, respectively). Further studies are warranted to investigate trastuzumab sensitivity in EOGC patients.

HER2 amplification and overexpression correlated in $92 \%$ of GC and discrepancies were mainly seen in CISH low-level amplified and IHC 2+ cases. Concordance percentages between IHC and FISH reported in literature range between 86.9 and $96.4 \%$ [16]. This study reports $7 / 180 \mathrm{IHC} / 1+(4 \%)$ cases to be CISH (low-level) amplified and four of the 9 (44\%) IHC 2+ cases to be CISH amplified. Our percentage of amplification in IHC $0 / 1+$ cases is similar to that of Bilous et al. (5.1\%) [4] but is in contrast to the results from the ToGA trial [1] that reported a higher frequency of IHC $0 / 1+$ samples testing FISH positive (22\%) compared to that in breast cancer.

In this TMA study, CISH showed less intratumoural heterogeneity than IHC, even after applying the additional scoring guideline, suggesting that $\mathrm{CISH}$ would be the preferable primary screening method for GC. Nevertheless, since this study did not use whole slides, this should be interpreted with caution. Furthermore, the ToGA trial reported that FISH positive but IHC $0 / 1+$ cases did not show a substantial overall survival benefit from trastuzumab (HR 1.07) compared to IHC $2+/$ FISH positive and IHC $3+$ /FISH negative cases (HR 0.65) [2], suggesting that, if this can be independently validated, IHC should be the primary screening method.

In conclusion, this study reports lower amplification and overexpression frequencies than previously described but confirms previous studies showing different frequencies depending on the type and localisation of the GC. Furthermore, we observed a lower amplification and overexpression frequency in EOGC and report a slightly lower heterogeneity rate by CISH compared to IHC.

Open Access This article is distributed under the terms of the Creative Commons Attribution Noncommercial License which permits any noncommercial use, distribution, and reproduction in any medium, provided the original author(s) and source are credited.

\section{References}

1. Y. Bang, H. Chung, J. Xu, F. Lordick, A. Sawaki, N. Al-Sakaff et al., Pathological features of advanced gastric cancer (GC): Relationship to human epidermal growth factor receptor 2 (HER2) positivity in the global screening programme of the ToGA trial. J. Clin. Oncol. 27, 4556 (2009)

2. Y.J. Bang, C.E. Van, A. Feyereislova, H.C. Chung, L. Shen, A. Sawaki et al., Trastuzumab in combination with chemotherapy versus chemotherapy alone for treatment of HER2-positive advanced gastric or gastro-oesophageal junction cancer (ToGA): a phase 3, open-label, randomised controlled trial. Lancet 376, 687-697 (2010)

3. J.D. Barros-Silva, D. Leitao, L. Afonso, J. Vieira, M. nis-Ribeiro, M. Fragoso et al., Association of ERBB2 gene status with histopathological parameters and disease-specific survival in gastric carcinoma patients. Br J Cancer 100, 487-493 (2009)

4. M.Bilous, R.Y.Osamura, J.Ruschoff, d.van, V, W.Hanna, F. Penault-Llorca et al., HER-2 amplification is highly homogenous in gastric cancer. Hum. Pathol. 41, 304-305 (2010)

5. A. Brufsky, Trastuzumab-based therapy for patients with HER2positive breast cancer: from early scientific development to foundation of care. Am. J. Clin. Oncol. 33, 186-195 (2010)

6. R. Carvalho, A.N. Milne, M. Polak, W.E. Corver, G.J. Offerhaus, M. A. Weterman, Exclusion of RUNX3 as a tumour-suppressor gene in early-onset gastric carcinomas. Oncogene 24, 8252-8258 (2005)

7. R. Carvalho, A.N. Milne, B.P. van Rees, E. Caspers, L. Cirnes, C. Figueiredo et al., Early-onset gastric carcinomas display molecular characteristics distinct from gastric carcinomas occurring at a later age. J Pathol 204, 75-83 (2004)

8. P. Correa, Y.H. Shiao, Phenotypic and genotypic events in gafstric carcinogenesis. Cancer Res. 54, 1941s-1943s (1994)

9. A.Dobi, G.Kelemen, L.Kaizer, R.Weiczner, L.Thurzo, and Z.Kahan, Breast Cancer under 40 Years of Age: Increasing Number and Worse Prognosis, Pathol Oncol Res (2010), doi:10.1007/s12253-0109305-3

10. H. Furukawa, T. Iwanaga, S. Imaoka, M. Hiratsuka, I. Fukuda, T. Kabuto et al., Multifocal gastric cancer in patients younger than 50 years of age. Eur. Surg. Res. 21, 313-318 (1989)

11. H. Grabsch, S. Sivakumar, S. Gray, H.E. Gabbert, W. Muller, HER2 expression in gastric cancer: Rare, heterogeneous and of no prognostic value - conclusions from 924 cases of two independent series. Cell. Oncol. 32, 57-65 (2010)

12. C. Gravalos, A. Jimeno, HER2 in gastric cancer: a new prognostic factor and a novel therapeutic target. Ann. Oncol. 19, 1523-1529 (2008)

13. S. Guiu, M. Gauthier, B. Coudert, F. Bonnetain, L. Favier, S. Ladoire et al., Pathological complete response and survival according to the level of HER-2 amplification after trastuzumabbased neoadjuvant therapy for breast cancer. Br. J. Cancer 103, 1335-1342 (2010)

14. K. Hede, Gastric cancer: trastuzumab trial results spur search for other targets. J. Natl Cancer Inst. 101, 1306-1307 (2009)

15. M. Hofmann, O. Stoss, D. Shi, R. Buttner, V.W. d.van Kim et al., Assessment of a HER2 scoring system for gastric cancer: results from a validation study. Histopathology 52, 797-805 (2008)

16. J.T. Jorgensen, Targeted HER2 treatment in advanced gastric cancer. Oncology 78, 26-33 (2010)

17. P.A. Kaufman, G. Broadwater, K. Lezon-Geyda, CALGB 150002: correlation of HER2 and chromosome 17 (ch17) copy number with trastuzumab (T) efficacy in CALGB 9840, paclitaxel (P) with or without $\mathrm{T}$ in HER2+ and HER2- metastatic breast cancer (MBC). J. Clin. Oncol. 25(Suppl 18), 1009 (2007)

18. D.Y. Kim, S.Y. Ryu, Y.J. Kim, S.K. Kim, Clinicopathological characteristics of gastric carcinoma in young patients. Langenbecks Arch. Surg. 388, 245-249 (2003) 
19. A. Kokkola, P. Sipponen, Gastric carcinoma in young adults. Hepatogastroenterology 48, 1552-1555 (2001)

20. P. Lauren, The two histological main types of gastric carcinoma: diffuse and so-called intestinal-type carcinoma. An attempt at a histo-clinical classification. Acta Pathol Microbiol Scand 64, 3149 (1965)

21. S. Lim, H.S. Lee, H.S. Kim, Y.I. Kim, W.H. Kim, Alteration of Ecadherin-mediated adhesion protein is common, but microsatellite instability is uncommon in young age gastric cancers. Histopathology 42, 128-136 (2003)

22. M. Maeta, H. Yamashiro, A. Oka, S. Tsujitani, M. Ikeguchi, N. Kaibara, Gastric cancer in the young, with special reference to 14 pregnancy-associated cases: analysis based on 2, 325 consecutive cases of gastric cancer. J. Surg. Oncol. 58, 191-195 (1995)

23. A.H. Marx, L. Tharun, J. Muth, A.M. Dancau, R. Simon, E. Yekebas et al., HER-2 amplification is highly homogenous in gastric cancer. Hum. Pathol. 40, 769-777 (2009)

24. P.J. Matley, D.M. Dent, M.V. Madden, S.K. Price, Gastric carcinoma in young adults. Ann. Surg. 208, 593-596 (1988)

25. A.N. Milne, F. Carneiro, C. O’Morain, G.J. Offerhaus, Nature meets nurture: molecular genetics of gastric cancer. Hum. Genet. 126, 615-628 (2009)

26. A.N. Milne, R. Carvalho, M. Jansen, E.K. Kranenbarg, C.J. van de Velde, F.M. Morsink et al., Cyclin E low molecular weight isoforms occur commonly in early-onset gastric cancer and independently predict survival. J Clin Pathol 61, 311-316 (2008)

27. A.N. Milne, R. Carvalho, F.M. Morsink, A.R. Musler, W.W. de Leng, A. Ristimaki et al., Early-onset gastric cancers have a different molecular expression profile than conventional gastric cancers. Mod Pathol 19, 564-572 (2006)

28. A.N. Milne, R. Leguit, W.E. Corver, F.H. Morsink, M. Polak, W. W. de Leng et al., Loss of CDC4/FBXW7 in gastric carinoma. Cell Oncol 32, 347-359 (2010)

29. A.N. Milne, R. Sitarz, R. Carvalho, F. Carneiro, G.J. Offerhaus, Early onset gastric cancer: on the road to unraveling gastric carcinogenesis. Curr. Mol. Med. 7, 15-28 (2007)

30. S. Paik, C. Kim, N. Wolmark, HER2 status and benefit from adjuvant trastuzumab in breast cancer. N. Engl. J. Med. 358, 1409-1411 (2008)

31. D.I. Park, J.W. Yun, J.H. Park, S.J. Oh, H.J. Kim, Y.K. Cho et al., HER-2/neu amplification is an independent prognostic factor in gastric cancer. Dig. Dis. Sci. 51, 1371-1379 (2006)

32. J.C. Park, Y.C. Lee, J.H. Kim, Y.J. Kim, S.K. Lee, W.J. Hyung et al., Clinicopathological aspects and prognostic value with respect to age: an analysis of 3, 362 consecutive gastric cancer patients. J. Surg. Oncol. 99, 395-401 (2009)

33. D.M. Parkin, F. Bray, J. Ferlay, P. Pisani, Estimating the world cancer burden: Globocan 2000. Int. J. Cancer 94, 153-156 (2001)
34. J.Ruschoff, M.Dietel, G.Baretton, S.Arbogast, A.Walch, G. Monges et al., HER2 diagnostics in gastric cancer-guideline validation and development of standardized immunohistochemical testing. Virchows Arch. 457, 299-307 (2010)

35. R. Santoro, F. Carboni, P. Lepiane, G.M. Ettorre, E. Santoro, Clinicopathological features and prognosis of gastric cancer in young European adults. Br. J. Surg. 94, 737-742 (2007)

36. R. Sitarz, R.J. Leguit, W.W. de Leng, F.H. Morsink, W.P. Polkowski, R. Maciejewski et al., Cyclooxygenase-2 mediated regulation of E-cadherin occurs in conventional but not earlyonset gastric cancer cell lines. Cell Oncol 31, 475-485 (2009)

37. B.R. Smith, B.E. Stabile, Extreme aggressiveness and lethality of gastric adenocarcinoma in the very young. Arch. Surg. 144, 506$510(2009)$

38. T. Takehana, K. Kunitomo, K. Kono, F. Kitahara, H. Iizuka, Y. Matsumoto et al., Status of c-erbB-2 in gastric adenocarcinoma: a comparative study of immunohistochemistry, fluorescence in situ hybridization and enzyme-linked immuno-sorbent assay. Int. J. Cancer 98, 833-837 (2002)

39. M. Tanner, M. Hollmen, T.T. Junttila, A.I. Kapanen, S. Tommola, Y. Soini et al., Amplification of HER-2 in gastric carcinoma: association with Topoisomerase IIalpha gene amplification, intestinal type, poor prognosis and sensitivity to trastuzumab. Ann. Oncol. 16, 273-278 (2005)

40. C.P.Theuer, V.C.de, G.Keese, S.French, T.Arnell, J.Tolmos et al., Gastric adenocarcinoma in patients 40 years of age or younger, Am J Surg 172 (1996), 473-476

41. S. Uchino, H. Tsuda, K. Maruyama, T. Kinoshita, M. Sasako, T. Saito et al., Overexpression of c-erbB-2 protein in gastric cancer. Its correlation with long-term survival of patients. Cancer 72, 3179-3184 (1993)

42. E. van Cutsem, Y. Kang, H. Chung, L. Shen, A. Sawaki, F. Lordick et al., Efficacy results from the ToGA trial: A phase III study of trastuzumab added to standard chemotherapy (CT) in first-line human epidermal growth factor receptor 2 (HER2)-positive advanced gastric cancer (GC). J. Clin. Oncol. 27, LBA4509 (2009)

43. B. Yan, E.X. Yau, S.S. Bte Omar, C.W. Ong, B. Pang, K.G. Yeoh et al., A study of HER2 gene amplification and protein expression in gastric cancer. J. Clin. Pathol. 63, 839-842 (2010)

44. T. Yano, T. Doi, A. Ohtsu, N. Boku, K. Hashizume, M. Nakanishi et al., Comparison of HER2 gene amplification assessed by fluorescence in situ hybridization and HER2 protein expression assessed by immunohistochemistry in gastric cancer. Oncol. Rep. 15, 65-71 (2006)

45. G.Z. Yu, Y. Chen, J.J. Wang, Overexpression of Grb2/HER2 signaling in Chinese gastric cancer: their relationship with clinicopathological parameters and prognostic significance. J. Cancer Res. Clin. Oncol. 135, 1331-1339 (2009) 